\title{
Construcción y validación de un instrumento para valorar desempeños pedagógicos de estudiantes en formación inicial
}

Aguilar Correa, Cristián Manuel; Sánchez Sánchez, Gerardo Ignacio

Construcción y validación de un instrumento para valorar desempeños pedagógicos de estudiantes en formación inicial

Revista Educación, vol. 42, núm. 1, 2018

Universidad de Costa Rica, Costa Rica

Disponible en: http://www.redalyc.org/articulo.oa?id=44051918006

DOI: https://dx.doi.org/10.15517/revedu.v42i1.22728

Esta obra está bajo una Licencia Creative Commons Atribución-NoComercial-SinDerivar 3.0 Internacional. 


\section{Construcción y validación de un instrumento para valorar desempeños pedagógicos de estudiantes en formación inicial}

\section{Construction and validation of an instrument to assess educational performance of students in initial training}

Cristián Manuel Aguilar Correa [1]

Universidad Católica del Maule, Chile

caguilar@ucm.cl

Gerardo Ignacio Sánchez Sánchez [2]

Universidad Católica del Maule, Chile

gsanchez@ucm.cl

\section{Resumen:}

La investigación tuvo por objetivo construir y validar un instrumento para valorar desempeños pedagógicos de estudiantes en formación inicial, a partir de los estándares para estudiantado egresado definidos por el Ministerio de Educación de Chile (2011). La validación fue desarrollada a partir de juicio de personas expertas con base en procedimiento planteado por los autores Rovinelli y Hambleton (1977). Posteriormente se aplicó el instrumento a una muestra probabilística de 58 estudiantes de Pedagogía Básica de la Universidad Católica del Maule (Chile), con el objeto de determinar su consistencia interna. Los resultados muestran valores aceptables en el índice de congruencia y factor de correspondencia, ya que la totalidad se ubica sobre el índice 0,40 . La consistencia interna promedió un índice total de 0.98. Los resultados muestran que el instrumento presenta adecuadas características psicométricas, respecto a la validez de contenido a través de personas expertas y la consistencia interna mediante el alfa de Cronbach.

Palabras ClaVE: Formación inicial docente, desempeños pedagógicos, estudiantes, validación.

\section{Abstract:}

This research aimed to construct and validate an instrument to assess educational performance of students in initial training from the graduates standards defined by the Ministerio de Educación de Chile (2011). The validation was developed from expert opinion based on the procedure proposed by the authors Rovinelli and Hambleton (1977). Later, the instrument was applied to a sample of 58 students of Basic Pedagogy at the Universidad Católica del Maule (Chile), in order to determine its internal consistency. The results showed acceptable values in the index of consistency and correspondence factor, since all are located on the index 0.40 . The internal consistency index averaged a total of 0.98 . The results show that the instrument presents adequate

\section{NotAS DE AUTOR}

[1] Profesor de Educación General Básica, Magister en Educación de las Ciencias y Doctor en Didáctica de las Ciencias Experimentales por la Universidad Complutense de Madrid. Posee más de 10 años de experiencia educativa en el sistema público escolar chileno. En el último tiempo ha trabajado formando personal docente futuro, inicialmente en la Escuela de Educación de la Universidad Católica del Norte (Antofagasta-Chile) y en la actualidad en la Facultad de Ciencias de la Educación, adscrito al Proyecto de mejoramiento institucional (PMI 1310) de la Universidad Católica del Maule-Chile. Su trabajo investigativo lo ha focalizado hacia temáticas de formación inicial docente (FID), didáctica de las ciencias naturales y educación ambiental. Algunas de sus publicaciones son: Aguilar, C. (2013). Estudio de las concepciones alternativas de los alumnos de enseñanza secundaria sobre conceptos estructurantes de ecología a través de la técnica de redes semánticas naturales. Estudios hemisféricos y polares; Aguilar, C. Brito, 1. (2011). La educación ambiental en la enseñanza formal obligatoria en Chile: Hacia una didáctica ambiental ecosistémica. Artículos y ensayos de sociología rural y Aguilar, C. (2017). La construcción del significado psicológico del concepto profesor o profesora por parte de grupos universitarios de estudiantes de pedagogía. Revista Electrónica Educare. http://www.revistas.una.ac.cr/index.php/EDUCARE/article/ view/7557/10416

[2] Profesor de Historia y Geografía. Magister en política y gestión educacional (Universidad de Talca). Doctor en Ciencias de la Educación (Universidad de la Serena). Académico adscrito al Proyecto de mejoramiento institucional (PMI 1310) de la Universidad Católica del Maule - CHILE. Posee una vasta experiencia en procesos de formación práctica y evaluación del aprendizaje en escuelas y liceos del sistema escolar municipal de la región del Maule, Chile. Ha publicado el texto "Aprender a enseñar situando las tareas del profesor". Ha dirigido múltiples investigaciones y expuesto en varios congresos nacionales. Algunas de sus publicaciones son: Visión del trabajo docente en el ámbito de la evaluación, que comienza a construir el profesorado en formación, a partir del uso de incidentes críticos en los procesos de formación práctica. Revista Electrónica Educare http://www.revistas.una.ac.cr/index.php/EDUCARE/article/view/6589/6691; Los espacios de tutoría en práctica profesional y sus necesidades de fortalecimiento. Revista Actualidades Investigativas en Educación http://revistas.ucr.ac.cr/index.php/aie/article/view/14807 y Visión del trabajo docente, de profesores en formación, a partir del uso de incidentes críticos. Revista Actualidades Investigativas en Educación. http://revistas.ucr.ac.cr/index.php/aie/article/view/20987 
psychometric characteristics, regarding the validity of content through expert people and the internal consistency through the Alfa of Cronbach.

KEYWORDs: Initial Teacher Training, Educational Performances, Students, Validation.

\section{INTRODUCCIÓN}

La sociedad moderna delega en la educación superior la función de desarrollar en sus estudiantes las habilidades que les permitan actuar de manera eficaz. "Esta actuación tiene dos dimensiones fundamentales, la profesional y la ciudadana. La adquisición de competencias se sitúa en el núcleo de esta demanda” (Yániz y Villardón, 2006, p. 13).

En Chile, la educación superior, sus políticas de financiamiento y de aseguramiento de la calidad, así como el aumento de la cobertura y la masificación en el acceso a ella, han generado nuevos desafíos y demandas, tanto para las distintas instituciones como para los actores involucrados en ellas. (Brunner, 2008)

Si bien en las políticas de aseguramiento de calidad como en los proyectos de mejoramiento de estas se observa que los procesos de enseñanza y aprendizaje, el perfeccionamiento docente y el fortalecimiento de sus prácticas son un tema central para el desarrollo de procesos educativos óptimos, el Informe de la OCDE sobre educación superior en Chile señala que no está del todo claro si se está logrando un mejoramiento real en esta área, lo cual se traduce en que "el elemento más débil en el marco de la calidad de la educación superior en Chile sigue siendo el aseguramiento y promoción de la calidad en la docencia de pregrado" (OCDE y BIRD/Banco Mundial, 2009, p. 192).

Este diagnóstico se complementa con el planteamiento de Brunner (2008) en términos de que "el aprendizaje continúa siendo definido como un proceso de adquisición de conocimientos, débilmente entrelazado con la práctica reflexiva que supone forma el núcleo de las profesiones” (p. 128).

En el último tiempo, se han desplegado relevantes esfuerzos para mejorar la calidad y equidad de la educación, planteándose, como prioridad, el fortalecimiento de la profesión docente, inicial y en servicio.

Dentro de las propuestas más significativas figuran: El Programa de Fortalecimiento de la Formación Inicial Docente (PFFID); la beca vocación de profesores; la definición de estándares de desempeño para la formación inicial docente; la acreditación obligatoria para las carreras de pedagogía desde el año 2006 y, lo más reciente INICIA (Evaluación Diagnóstica de la Evaluación Inicial Docente).

El PFIDD (1997-2001), orientado a la reforma de los proyectos curriculares de las carreras, releva la centralidad de una formación práctica temprana y progresiva. El proceso de acreditación de las carreras de pedagogía que entró en vigencia en noviembre de 2006 con carácter prescriptivo y orientado a introducir mecanismos de autorregulación. La Prueba INICIA, entendida como examen de excelencia profesional con el propósito de evaluar las competencias de egreso del profesorado, en la actualidad está transitando a una evaluación más bien diagnóstica orientada a proveer información a las instituciones de formación inicial, para que puedan retroalimentar sus proyectos curriculares. Por otro lado, en un esfuerzo por atraer mayores talentos a pedagogía, surge la Beca Vocación de Profesor (2011).

Probablemente lo que ha constituido un avance para enfrentar los desafíos de calidad esperables de la formación inicial es la elaboración de estándares orientadores para las carreras de pedagogía a partir del año 2011, con énfasis en el estudiantado egresado de educación general básica, para, desde ahí, avanzar a las pedagogías de enseñanza media y educación parvularia. Los estándares tienen por función servir de orientaciones a las carreras de pedagogía estableciendo los conocimientos y habilidades que su estudiantado egresado debe demostrar al enseñar. Es así como los estándares "señalan un 'qué, referido a un conjunto de aspectos o dimensiones que se debieran observar en el desempeño de un futuro profesor o profesora; y también, establecen un 'cuánto' o medida, que permite evaluar lejos o cerca se encuentra un nuevo profesor o profesora de alcanzar un determinado desempeño" (Ministerio de Educación, 2011, p. 7). 
Estos estándares contemplan la definición de los contenidos, las habilidades y las actitudes profesionales esperables de un sujeto egresado de pedagogía. En esta dirección, dichos estándares serán un referente para los procesos de evaluación de personas egresadas y responde a necesidades diversas: tomar decisiones, mejorar la formación, responder a la demanda social de responsabilidad, favorecer la profesionalización del profesorado y certificar competencias (Yániz y Villardón, 2006).

En ese escenario, la presente investigación tiene como objetivo construir y validar un instrumento que permita valorar los desempeños pedagógicos que movilizan estudiantes en formación inicial, en sus contextos de inserción.

\section{Marco té́rico}

\section{La evaluación del desempeño docente}

La evaluación tiene diversos sentidos y utilidades; también puede ser vista en distintos ámbitos de acción, en este caso la podemos reconocer como una herramienta que permite dar cuenta de los aciertos y desaciertos del desempeño profesional, y es de utilidad, si permite la retroalimentación para el mejoramiento del futuro profesorado.

Los modelos de evaluación se reconocen según el fin que persiguen y por lo tanto son altamente consistentes con la comprensión del proceso en el que se insertan. El "logro de metas", por ejemplo, asociado curricularmente a una docencia basada en objetivos, es promovida por Tyler, (1986), quien desarrolla el primer método sistemático de evaluación educacional orientado a la búsqueda de la congruencia.

La evaluación que centra su interés en el juicio y el criterio, planteada entre otros estudios por Stake (1967), se sustenta en la contratación de la información desde el punto de vista descriptivo y valorativo. Este enfoque se acerca más al uso del criterio como elemento de expectativas compartidas. Complementariamente, desde la perspectiva de la evaluación debe orientarse al perfeccionamiento (proceso), es decir, "La razón de ser de la evaluación es contribuir claramente a mejorar el objeto evaluado, incidiendo, como habría de esperarse, en la toma de decisiones" (Pimienta, 2008, p.14). Tengamos presente que el modelo planteado por el autor está integrado por el contexto, entrada, proceso y producto; todo orientado a la toma de decisiones y al mejoramiento. Así, los aspectos claves del objeto que deben valorarse incluyen sus metas, su planificación, su realización y su impacto. El aporte mayor de este uso se relaciona con que, si la evaluación no sirve para mejorar, probablemente no es necesaria su implementación.

$\mathrm{Al}$ respecto, es importante señalar el aporte de Tobón, (2007, p. 15), quien se refiere al sentido de la evaluación entendida en un marco de gestión de calidad. Desde allí propone las bondades de la definición de competencias en un enfoque complejo:

1. las competencias se abordan desde el proyecto ético de vida de las personas, para afianzar la unidad e identidad de cada ser humano, y no su fragmentación; 2) las competencias buscan reforzar y contribuir a que las personas sean emprendedoras, primero como seres humanos y en la sociedad, y después en lo laboral-empresarial para mejorar y transformar la realidad;3) las competencias se abordan en los procesos formativos desde unos fines claros, socializados, compartidos y asumidos en la institución educativa, que brinden un para qué que oriente las actividades de aprendizaje, enseñanza y evaluación; 4) la formación de competencias se da desde el desarrollo y fortalecimiento de habilidades de pensamiento complejo como clave para formar personas éticas, emprendedoras y competentes; y 5) desde el enfoque complejo la educación no se reduce exclusivamente a formar competencias, sino que apunta a formar personas integrales, con sentido de la vida, expresión artística, espiritualidad, conciencia de sí, etc., y valores. 
Considerando distintas fuentes de información que se aproximan a la cuestión de la evaluación del desempeño docente, el estudio comparado de UNESCO (2004) aporta una mirada desde la concepción de la evaluación que es importante relevar en cualquier aproximación a ello. Desde una visión muy tradicional centrada en la verificación de estándares mínimos, por parte del personal docente, se ha avanzado a una visión más de proceso, vinculada al desempeño en contextos; asumiendo una mirada más formativa orientada al desarrollo profesional.

\section{La formación inicial y el desempeño docente}

La formación inicial es entendida como el proceso de entrega y apropiación de un saber científico estandarizado, que tiene lugar en una institución especializada en esa tarea, durante un lapso explícito de tiempo y que es certificado en la forma de habilidades y disposiciones adquiridas por estudiantes o profesionales en formación (Gyslin, 1992).

Lo anterior cobra relevancia, si se considera que la formación inicial explica o debería explicar la calidad del quehacer del profesorado en formación, lo cual se pone a prueba en los procesos de práctica e inserción profesional (Fuentealba, 2003). Y que deben permitir concebir al cuerpo docente como profesional de la articulación del proceso de enseñanza - aprendizaje en situación (Paquay, Altet, Charlier y Perrenoud, 2010). Para su aprendizaje, el futuro profesorado se despliega en dos niveles de realidad: uno que tiene relación con su condición de personal docente activo, en el aula, y una segunda dimensión en donde reflexiona sobre esa práctica (Galaz, Fuentealba, Cornejo y Padilla, 2011).

Un aspecto central de los últimos años sobre formación de profesorado se refiere a cierto consenso especializado en relación con la complejidad en niveles cognitivos, prácticos y afectivos para el desempeño del profesorado (Calderhead y Shorrock, 1997).

La máxima difundida en el último tiempo, respaldada por la investigación, sobre que un buen profesorado genera más y mejores aprendizajes, ha despertado el interés en identificar cuáles son las características de ese personal docente efectivo. Por ello, diversos países, incluido Chile, han dado relevancia a los estándares sobre desempeño docente, entendidos como referentes de calidad para la enseñanza. En este contexto, los estándares de desempeño docente resultan un instrumento pertinente, ya que representan conocimientos, aptitudes, opiniones y disposiciones de aquel profesorado eficaz que puede ofrecer una enseñanza de calidad. Además, este tipo de estándares logra describir explícitamente las características y tareas de la enseñanza docente.

Es importante constatar que los estándares o competencias permiten definir un conjunto observable de prácticas profesionales o actuaciones profesionales integrando saberes, habilidades y destrezas, las actitudes y valores para dar respuesta a los retos profesionales contextualizados con el fin de promover el desarrollo profesional.

Para el caso de la formación de profesorado, los planteamientos de Saravia (2009) y Perrenoud (2006) permiten aproximarnos a las competencias docentes. Saravia (2009) define la competencia profesional del profesorado como:

Conjunto de cualidades internas que le permitan sostener y aplicar un discurso científico desde el cual generar procesos de aprendizaje permanente, en sentido personal y grupal, con visión innovadora hacia un desarrollo proactivo e integral de su profesionalidad. (p. 18)

La propuesta de Saravia (2009) considera cuatro conjuntos o categorías de competencias: la científica, práctica, personal y, finalmente, la social. En cada una de ellas identifica y releva dimensiones e indicadores. Desde su perspectiva, la competencia científica alude al conjunto de conocimientos teóricos contrastados empíricamente, que permiten a la persona entender partes concretas de la realidad, interpretar su sentido y definir posibilidades de intervención; la competencia práctica, referida al conjunto de conocimientos 
metodológicos sistematizados y de procedimientos empíricos naturales que permiten a la persona actuar correctamente en partes definidas de la realidad; la competencia personal, entendida como el conglomerado de cualidades que permiten a la persona tener conciencia clara de la realidad para regular su desempeño en la vida, a partir de valores éticos convencionales de impacto; $y$, muy vinculada a la anterior, reconoce la presencia de una competencia social que vincula las cualidades que permiten a la persona una correcta y enriquecedora relación con su contexto social y natural, según valores sociales de convivencia armónica entre las personas y su entorno (Saravia, 2009).

En el caso de Perrenoud (2006), formar competencias reales durante la formación supone una transformación considerable de la relación del profesorado con el saber, de sus maneras de "hacer clases" y, a fin de cuentas, de su identidad y de sus propias competencias profesionales.

Se puede considerar que nos encaminamos hacia una nueva profesión, en la cual el desafío es hacer aprender más que enseñar Los nuevos enfoques añaden a las exigencias de la centralidad en el alumnado, la pedagogía diferenciada y los métodos activos y la necesidad de:

- Considerar los saberes como recursos para movilizar.

- Trabajar regularmente a través de problemas.

- Crear o utilizar otros medios de enseñanza.

- Negociar y conducir proyectos con sus estudiantes.

- Adoptar una planificación flexible e indicativa.

- Establecer y explicitar un nuevo contrato didáctico.

- Practicar una evaluación formadora en situaciones de trabajo.

- Dirigirse hacia una menor separación disciplinaria (Perrenoud, 2006, p. 69).

En relación con esta última condición, se sugiere que los cuerpos docentes, por muy especializados que sean,

se sientan responsables de la formación global de cada alumno más que exclusivamente responsables de sus conocimientos en su propia disciplina; que aprovechen la menor ocasión para salir de su campo de especialización y discutan, con sus colegas, problemas de método, de epistemología, relacionados con el saber, con la investigación; que perciban y valoricen las transversalidades potenciales, en los programas y en las actividades didácticas. (Perrenoud, 2006, p. 88)

Surge así el requerimiento de un nuevo tipo de profesionalidad, de identidad, de formación en el trabajo docente.

Disponer de estándares para la formación de docentes permite desarrollar una visión común de las habilidades profesionales, en nuestro caso entendidas como el conjunto de conocimientos, procedimientos y el saber - estar, pero también el hacer y el ser necesarios para el ejercicio de la profesión docente. Entendiendo, según Anderson (1986, p. 41, citado por Paquay, et al. 2010), "que estas habilidades son de orden cognitivo, afectivo, conativo y práctico. Así mismo, éstas son dobles: de orden técnico y didáctico en cuanto a la preparación de los contenidos, pero también de orden relacional, pedagógico y social en cuanto a la adaptación a las interacciones en clase”. De acuerdo con Imbernón (2001), el enseñar constituye un proceso que trasciende el ámbito meramente técnico para alcanzar el ámbito personal, profesional y social.

$\mathrm{Al}$ revisar los documentos oficiales, el Ministerio de Educación, el año 2011, a través de la elaboración de Estándares orientadores para egresados de carreras de pedagogía, busca asegurar en el estudiantado egresado:

Habilidades y actitudes personales tales como capacidad de trabajo colaborativo, autonomía, flexibilidad, capacidad de innovar, disposición al cambio y proactividad. Al mismo tiempo y como complemento se espera que tenga una sólida formación en valores, y presente un comportamiento ético adecuado. Además, tiene que ser capaz de comunicarse por escrito y oralmente en forma eficaz en su lengua original y en una segunda lengua. Por otra parte, se espera que el futuro profesional demuestre habilidades en el uso de tecnologías de la información y la comunicación.

De igual forma, deberá poseer un comportamiento que refleje un nivel cultural adecuado a las exigencias de un mundo globalizado. Del mismo modo, debe mostrar un espíritu de superación personal y profesional, por lo cual debe tener conciencia del desarrollo profesional como una constante de vida. (p.19) 
Estos estándares vienen a dialogar con otro instrumento técnico orientador de la gestión del aula, denominado Marco para la buena enseñanza (MINEDUC-CPEIP, 2008) el cual supone que:

Los profesionales que se desempeñan en las aulas, antes que nada, son educadores comprometidos con la formación de sus estudiantes. Supone que, para lograr la buena enseñanza, los docentes se involucran como personas en la tarea, con todas sus capacidades y sus valores. (p. 7)

Desde otra perspectiva:

Reconoce la complejidad de los procesos de enseñanza y aprendizaje y los variados contextos culturales en que éstos ocurren, tomando en cuenta las necesidades de desarrollo de conocimientos y competencias por parte de los docentes, tanto en materias a ser aprendidas como en estrategias para enseñarlas; la generación de ambientes propicios para el aprendizaje de todos sus alumnos; como la responsabilidad de los docentes sobre el mejoramiento de los logros estudiantiles. (MINEDUCCPEIP, 2008, p. 7).

Al mostrarse de acuerdo con la complejidad de la tarea docente, este marco desafía la formación inicial planteando responsabilidades considerables a los proyectos formativos de cada carrera. Dentro de esas responsabilidades, la evaluación pasa a ocupa un lugar prioritario.

\section{Procedimientos metodológicos}

\section{Participantes}

Para llevar a cabo el proceso de validación del instrumento fueron consultados cinco personas expertas en el tema, quienes evaluaron los ítems, en respuesta Likert, con el procedimiento planteado por los autores Rovinelli y Hambleton (1977). Posteriormente se inició el proceso de recolección de datos con el fin de poder analizar un análisis de confiabilidad mediante consistencia interna alfa de Cronbach. Este se realizó a través de una encuesta de autorreporte en línea y se aplicó a una muestra no probabilística por conveniencia de 58 estudiantes de quinto año de las carreras de Pedagogía General Básica y Pedagogía General Básica con Mención, de la Universidad Católica del Maule (Chile). Este valor equivale al 53,2\% del total de un universo de 109 estudiantes de ambas carreras.

\section{Diseño}

El diseño utilizado fue no experimental, transeccional descriptivo, (Hernández, Fernández, Baptista, 2010) fundamentado desde una metodología de investigación de naturaleza cuantitativa.

\section{Procedimiento e instrumentos}

La construcción del instrumento contempló tres etapas. a) hicimos una exhaustiva revisión de los estándares orientadores para estudiantado egresado de carreras de Pedagogía en Educación Básica (Ministerio de Educación, 2011) y creamos 50 ítems, de respuesta Likert, conforme a definiciones conceptuales referidas a cinco dimensiones de la docencia: currículo, metodología, ambiente para el aprendizaje, evaluación y reflexión (García y Parra, 2010; Day, 2006; MINEDUC-CPEIP, 2008; Yániz y Villardón, 2006; Zabalza, 2003)

Currículo: entendido como proyecto formativo integrado (Zabalza, 2003) que explicita propósitos, fundamentos, estructura y secuencia de los diversos componentes de la planificación, tales como objetivos y su progresión, contenidos, actividades, orientaciones metodológicas y evaluativas desde las cuales el profesorado 
diseña propuestas pedagógicas, con capacidad de analizar y fundamentar su coherencia e impacto en el aprendizaje de sus estudiantes.

Además, mide cuán familiarizado se percibe a sí mismo el personal docente respecto a instrumentos oficiales tales como el marco curricular vigente, los programas de estudio y los estándares de aprendizaje definidos para cada nivel educativo.

Metodología: referida a la capacidad de actuar de una manera ordenada con el fin de alcanzar una meta, vale decir, la forma de actuación que se sigue en el aula para llevar a cabo el proceso de enseñanza del profesorado tendiente a hacer efectivo el aprendizaje del estudiantado (García y Parra, 2010). Desde el punto de vista didáctico, supone la definición de un proyecto de actuación que, a partir de los objetivos de aprendizaje declarados, articule elementos afectivos, cognitivos, experienciales y el contexto en que se desarrollará la docencia.

Ambiente para el aprendizaje: referido a la capacidad del personal docente de establecer un ambiente y clima, en el cual tiene lugar el proceso de enseñanza y que se relaciona con componentes sociales, afectivos y materiales del aprendizaje. En este ambiente preparado, la confianza, aceptación, equidad y respeto entre las personas se sustenta en normas constructivas de comportamiento y altas expectativas sobre las posibilidades de aprendizaje y desarrollo de todo el alumnado (MINEDUC-CPEIP, 2008) Evaluación: como elemento significativo de la acción didáctica supone un proceso sistemático de obtención de evidencia del aprendizaje, "con el objeto no sólo de constatar su aplicación, desarrollo y resultados, sino, sobre todo, con la finalidad de mejorarlos, ya que los procesos de enseñanza y aprendizaje son, en principio, perfectibles" (Castillo, 2002, p. 13). Implica la capacidad para tomar decisiones de mejora permanente orientadas a adaptar la planificación, ayudar al estudiantado a tomar conciencia de su punto de partida; asegurar la regulación continua del aprendizaje y certificar competencias. Constituye una guía de lo que se requiere aprender y facilita, además, la reflexión necesaria para introducir mejoras en el proceso (Yániz y Villardón, 2006).

Reflexión: Entendida como responsabilidad de la profesión, en función de la cual se indaga de manera consciente y sistemática sobre la práctica pedagógica desarrollada, y se procede a reformularla para contribuir al aprendizaje estudiantil, y al desarrollo profesional en un proceso de actualización de carácter permanente que lo vincula y compromete con la comunidad educativa de la que forma parte. "La reflexión implica una crítica de la práctica, los valores implícitos en esa práctica, los contextos normativos personales, sociales, institucionales y generales en los que tiene lugar la práctica, y las consecuencias para la mejora de esa práctica" (Day, 2016, p. 125).

b) Luego, los ítems elaborados fueron entregados a un grupo de cinco personas expertas quienes los evaluaron con base en el método planteado por Rovinelli y Hambleton (1977). Este procedimiento consistió en pedirles al grupo de jueces que evaluaran los ítems para todas las dimensiones del instrumento, asignándoles tres posibles calificaciones: no lo representa, lo representa medianamente o lo representa completamente. Posteriormente, estas calificaciones llevadas a -1, 0 y 1 respectivamente, permitirían calcular el índice de congruencia, el cual evalúa no solo si el ítem representa adecuadamente la dimensión que le corresponde, sino también, que tan inadecuadamente representa a los ítems para los que no está diseñado. Este indicador va de -1 a 1 y mientras más cercano a 1 sea el valor, más adecuado es el ítem para su dimensión.

c) Finalmente, acabada la evaluación de los ítems, se inició el proceso de recolección de datos con el fin de poder analizar las propiedades métricas del instrumento. Este procedimiento se llevó a cabo por medio de una encuesta de auto-reporte, en formato Google Docs, respondida por una muestra de 58 estudiantes pertenecientes a las carreras de Pedagogía General Básica y Pedagogía General Básica con mención de la Universidad Católica del Maule. Con base en los datos arrojados se realizó un análisis de confiabilidad mediante consistencia interna calculado con el alfa de Cronbach. Cabe señalar que este valor fluctúa entre 0 y 1 , en donde más cercano a 1 , mejor será la consistencia interna del instrumento. 


\section{ANÁLISIS Y DISCUSIÓN DE RESULTADOS}

\section{A. Dimensiones de la docencia e ítems}

A partir de las dimensiones de la docencia que fueron consideradas: currículo, metodología, ambiente para el aprendizaje, evaluación y reflexión. Se formularon los siguientes ítems:

Currículo:

- Domino conceptos básicos de teoría curricular.

- Domino los fundamentos pedagógicos sobre los cuales se ha construido el currículo de mi especialidad.

- Conozco los propósitos formativos que establece el currículo nacional para el nivel educativo de mi especialidad.

- Estoy preparado(a) para planificar el proceso de enseñanza comprometiendo a todo el estudiantado con los aprendizajes.

- Estoy preparado(a) para diseñar actividades atendiendo a las características de mis estudiantes según niveles y áreas curriculares.

- Integro los aprendizajes de las distintas áreas del currículo, reconociendo las relaciones de interdependencia entre ellas.

- Estoy preparado(a) para planificar el proceso de enseñanza-aprendizaje en diferentes contextos socioeducativos.

- Estoy preparado(a) para fundamentar las decisiones pedagógicas que adopto en mi planificación educativa.

- Domino los contenidos de las disciplinas de mi especialidad.

Metodología:

- Comunico en forma clara y precisa los objetivos de aprendizaje.

- Estoy preparado(a) para promover en mis estudiantes el desarrollo de habilidades de pensamiento superior.

- Abordo con rigurosidad conceptual el contenido de la clase asegurando su comprensión por parte de mis estudiantes.

- Genero estrategias de enseñanza desafiantes para el aprendizaje de mis estudiantes según contexto.

- Utilizo criterios pedagógicos congruentes con los principios y conceptos centrales de las asignaturas a enseñar.

- Utilizo las TIC para apoyar el desarrollo de la enseñanza concordante con los objetivos planificados.

- Valoro el error como fuente de información para que mis estudiantes mejoren sus aprendizajes.

- Sé cómo promover en el alumnado la comprensión del sentido de las actividades educativas.

Ambiente para el aprendizaje:

- Sé cómo promover en el alumnado la comprensión del sentido de las actividades educativas.

- Sé cómo generar espacios acogedores y estimulantes para un aprendizaje integral de todos mis estudiantes.

- Estoy preparado(a) para establecer normas de convivencia basadas en la tolerancia y el respeto mutuo.

- Estoy preparado(a) para gestionar el tiempo de la clase en favor de los objetivos de aprendizaje planificados. 
- Genero un ambiente organizado de trabajo disponiendo espacios y recursos en función de los aprendizajes.

- Estoy preparado(a) para favorecer un aula inclusiva, que atiende la diversidad estudiantil.

- Conozco estrategias para el manejo conductual de cursos numerosos.

- Formulo preguntas que estimulen a mis estudiantes a pensar y no solo reproducir contenidos.

- Genero y comunico el sentido de normas explicitas de convivencia.

- Utilizo estrategias de comunicación efectivas en el aula.

- Estructuro adecuadamente el espacio físico para la fluidez del trabajo pedagógico.

Evaluación:

- Valoro la evaluación diagnóstica para adaptar la planificación a las necesidades de mis estudiantes.

- Estoy preparado(a) para elaborar instrumentos evaluativos en función de los objetivos de aprendizaje planificados.

- Comunico en forma apropiada y oportuna a mis estudiantes los objetivos de aprendizaje.

- Monitoreo el proceso de comprensión y apropiación del aprendizaje por parte de mis estudiantes.

- Estoy preparado(a) para utilizar los resultados de la evaluación educativa con el objeto de retroalimentar el aprendizaje de mis estudiantes.

- Utilizo la información que proporciona la evaluación para identificar fortalezas y debilidades en mi enseñanza.

- Comunico en forma apropiada y oportuna a mis estudiantes los criterios de evaluación.

- Sé cómo retroalimentar a mis estudiantes acerca de sus avances académicos.

- Utilizo la evaluación diferenciada para dar cuenta de los aprendizajes en el espacio del aula.

- Selecciono estrategias e instrumentos de evaluación en función del tipo de aprendizaje a lograr (conceptuales, procedimentales o actitudinales).

Reflexión:

- Identifico mis fortalezas y debilidades, en relación con las disciplinas que enseño.

- Sé cómo construir relaciones de trabajo colaborativo con mis colegas.

- Estoy preparado(a) para introducir cambios en la mejorara de mi práctica pedagógica en función de los resultados de aprendizaje obtenidos.

- Analizo críticamente mi práctica pedagógica en función del impacto en el aprendizaje de mis estudiantes.

- Tengo una sólida formación ética que me permite estar consciente de mi responsabilidad en el desarrollo personal de mis estudiantes.

- Tengo una sólida formación ética que me permite estar consciente de mi responsabilidad en el desarrollo académico de mis estudiantes.

- Estoy preparado(a) para ser evaluado(a) en mi quehacer pedagógico al interior del aula

- Conozco los marcos nacionales que regulan el desempeño y la evaluación de la profesión docente.

- Manejo información actualizada sobre mi profesión, el sistema educativo y las políticas vigentes.

- Reconozco mis necesidades de desarrollo y actualización profesional.

\section{B. Validación por personas expertas}

Elaborado el instrumento, este fue sometido a validación de juicio de expertos con base en el método planteado por Rovinelli y Hambleton (1977). En la Tabla 1, se muestran los Ítems seleccionados para la aplicación final a la muestra, los que obtuvieron un índice de 0,40 hacia arriba. 
TABLA 1

Ítems considerados para la aplicación de la muestra

\begin{tabular}{|c|c|c|}
\hline & Ítem & $\begin{array}{c}\text { Indice de } \\
\text { concordancia }\end{array}$ \\
\hline \multirow[t]{5}{*}{ Currículo } & Domino conceptos básicos de teoría curricular & 0,68 \\
\hline & $\begin{array}{l}\text { Conozco los propósitos formativos que establece el currículo nacional para el } \\
\text { nivel educativo de mi especialidad }\end{array}$ & 0,53 \\
\hline & Domino los contenidos de las disciplinas de mi especialidad & 0,63 \\
\hline & $\begin{array}{l}\text { Domino los fundamentos pedagógicos sobre los cuales se ha construido el } \\
\text { currículo de mi especialidad }\end{array}$ & 0,48 \\
\hline & $\begin{array}{l}\text { Estoy preparado(a) para planificar el proceso de enseñanza-aprendizaje en } \\
\text { diferentes contextos socioeducativos }\end{array}$ & 0,45 \\
\hline \multirow[t]{4}{*}{ Didáctica } & $\begin{array}{l}\text { Utilizo las TIC para apoyar el desarrollo de la enseñanza de acuerdo con los } \\
\text { objetivos planificados }\end{array}$ & 0,68 \\
\hline & $\begin{array}{l}\text { Manejo variadas estrategias de enseñanza-aprendizaje congruentes con la } \\
\text { naturaleza de los objetivos planificados }\end{array}$ & 0,45 \\
\hline & $\begin{array}{l}\text { Genero estrategias de enseñanza desafiantes para el aprendizaje de mis es- } \\
\text { tudiantes según contexto }\end{array}$ & 0,48 \\
\hline & $\begin{array}{l}\text { Utilizo criterios pedagógicos congruentes con los principios y conceptos cen- } \\
\text { trales de las asignaturas a enseñar }\end{array}$ & 0,43 \\
\hline \multirow{8}{*}{$\begin{array}{l}\text { Ambiente } \\
\text { para el } \\
\text { aprendizaje }\end{array}$} & $\begin{array}{l}\text { Sé cómo promover en el alumnado la comprensión del sentido de las activi- } \\
\text { dades educativas }\end{array}$ & 0,50 \\
\hline & $\begin{array}{l}\text { Sé cómo generar espacios acogedores y estimulantes para un aprendizaje } \\
\text { integral de todo el estudiantado }\end{array}$ & 0,55 \\
\hline & $\begin{array}{l}\text { Estoy preparado(a) para establecer normas de convivencia basadas en la } \\
\text { tolerancia y el respeto mutuo }\end{array}$ & 0,58 \\
\hline & Conozco estrategias para el manejo conductual de cursos numerosos & 0,63 \\
\hline & $\begin{array}{l}\text { Formulo preguntas que estimulen a mis estudiantes a pensar y no solo re- } \\
\text { producir contenidos }\end{array}$ & 0,53 \\
\hline & Genero y comunico el sentido de normas explicitas de convivencia & 0,53 \\
\hline & Utilizo estrategias de comunicación efectivas en el aula & 0,55 \\
\hline & Estructuro adecuadamente el espacio físico para la fluidez del trabajo pedagógico & 0,50 \\
\hline \multirow[t]{6}{*}{ Evaluación } & $\begin{array}{l}\text { Monitoreo el proceso de comprensión y apropiación del aprendizaje por parte } \\
\text { de mis estudiantes }\end{array}$ & 0,50 \\
\hline & $\begin{array}{l}\text { Utilizo la información que proporciona la evaluación para identificar } \\
\text { fortalezas y debilidades en mi enseñanza }\end{array}$ & 0,60 \\
\hline & $\begin{array}{l}\text { Comunico en forma apropiada y oportuna a mis estudiantes los criterios de } \\
\text { evaluación }\end{array}$ & 0,58 \\
\hline & $\begin{array}{l}\text { Valoro la evaluación diagnóstica para adaptar la planificación a las } \\
\text { necesidades de mis estudiantes }\end{array}$ & 0,45 \\
\hline & $\begin{array}{l}\text { Estoy preparado(a) para utilizar los resultados de la evaluación educativa } \\
\text { con el objeto de retroalimentar el aprendizaje de mis estudiantes }\end{array}$ & 0,40 \\
\hline & $\begin{array}{l}\text { Selecciono estrategias e instrumentos de evaluación en función del tipo de } \\
\text { aprendizaje a lograr (conceptuales, procedimentales o actitudinales) }\end{array}$ & 0,45 \\
\hline \multirow[t]{10}{*}{ Reflexión } & $\begin{array}{l}\text { Identifico mis fortalezas y debilidades, en relación con las disciplinas que } \\
\text { enseño }\end{array}$ & 0,63 \\
\hline & Sé cómo construir relaciones de trabajo colaborativo con mis colegas & 0,68 \\
\hline & $\begin{array}{l}\text { Estoy preparado(a) para introducir cambios en la mejorara de mi práctica } \\
\text { pedagógica en función de los resultados de aprendizaje obtenidos }\end{array}$ & 0,70 \\
\hline & $\begin{array}{l}\text { Analizo críticamente mi práctica pedagógica en función del impacto en el } \\
\text { aprendizaje de mis estudiantes }\end{array}$ & 0,75 \\
\hline & $\begin{array}{l}\text { Tengo una sólida formación ética que me permite estar consciente de mi re- } \\
\text { sponsabilidad en el desarrollo personal de mis estudiantes }\end{array}$ & 0,78 \\
\hline & $\begin{array}{l}\text { Tengo una sólida formación ética que me permite estar consciente de mi re- } \\
\text { sponsabilidad en el desarrollo académico de mis estudiantes }\end{array}$ & 0,75 \\
\hline & $\begin{array}{l}\text { Estoy preparado(a) para ser evaluado(a) en mi quehacer pedagógico al inte- } \\
\text { rior del aula }\end{array}$ & 0,63 \\
\hline & $\begin{array}{l}\text { Conozco los marcos nacionales que regulan el desempeño y la evaluación de } \\
\text { la profesión docente }\end{array}$ & 0,65 \\
\hline & $\begin{array}{l}\text { Manejo información actualizada sobre mi profesión, el sistema educativo y } \\
\text { las políticas vigentes }\end{array}$ & 0,83 \\
\hline & Reconozco mis necesidades de desarrollo y actualización profesional & 0,88 \\
\hline
\end{tabular}

\section{Análisis de confiabilidad mediante consistencia interna}

Con la participación del estudiantado que respondió el instrumento, se procedió a determinar el alfa de Cronbach. En la Tabla 2 se muestra dicho valor del instrumento total y por dimensiones. 
TABLA 2

Valores del alfa de Cronbach en el instrumento

\begin{tabular}{cc}
\hline Escala & Alpha de Cronbach \\
\hline Escala total & 0,98 \\
Currículo & 0,92 \\
Metodología & 0,92 \\
Ambiente para el aprendizaje & 0,95 \\
Evaluación & 0,94 \\
Reflexión & 0,95 \\
\hline
\end{tabular}

A continuación, la Tabla 3 muestra el alfa de Cronbach si cada ítem del instrumento fuera eliminado. 
TABLA 3

Alfa de Cronbach si cada ítem del instrumento fuera eliminado

\begin{tabular}{|c|c|}
\hline Ítem & $\begin{array}{l}\text { Alfa de Cronbach si el } \\
\text { elemento se ha suprimido }\end{array}$ \\
\hline Domino conceptos básicos de teoría curricular & 0,986 \\
\hline $\begin{array}{l}\text { Conozco los propósitos formativos que establece el currículo nacional para el } \\
\text { nivel educativo de mi especialidad }\end{array}$ & 0,987 \\
\hline Domino los contenidos de las disciplinas de mi especialidad & 0,986 \\
\hline $\begin{array}{l}\text { Domino los fundamentos pedagógicos sobre los cuales se ha construido el } \\
\text { currículo de mi especialidad }\end{array}$ & 0,987 \\
\hline $\begin{array}{l}\text { Estoy preparado(a) para planificar el proceso de enseñanza-aprendizaje en } \\
\text { diferentes contextos socioeducativos }\end{array}$ & 0,987 \\
\hline $\begin{array}{l}\text { Utilizo las TIC para apoyar el desarrollo de la enseñanza de acuerdo con los } \\
\text { objetivos planificados }\end{array}$ & 0,987 \\
\hline $\begin{array}{l}\text { Manejo variadas estrategias de enseñanza-aprendizaje congruentes con la } \\
\text { naturaleza de los objetivos planificados }\end{array}$ & 0,987 \\
\hline $\begin{array}{l}\text { Genero estrategias de enseñanza desafiantes para el aprendizaje de mis } \\
\text { estudiantes según contexto }\end{array}$ & 0,986 \\
\hline $\begin{array}{l}\text { Utilizo criterios pedagógicos congruentes con los principios y conceptos } \\
\text { centrales de las asignaturas a enseñar }\end{array}$ & 0,986 \\
\hline $\begin{array}{l}\text { Sé cómo promover en el alumnado la comprensión del sentido de las actividades } \\
\text { educativas }\end{array}$ & 0,987 \\
\hline $\begin{array}{l}\text { Sé cómo generar espacios acogedores y estimulantes para un aprendizaje } \\
\text { integral de todo el estudiantado }\end{array}$ & 0,986 \\
\hline $\begin{array}{l}\text { Estoy preparado(a) para establecer normas de convivencia basadas en la } \\
\text { tolerancia y el respeto mutuo }\end{array}$ & 0,987 \\
\hline Conozco estrategias para el manejo conductual de cursos numerosos & 0,987 \\
\hline $\begin{array}{l}\text { Formulo preguntas que estimulen a mis estudiantes a pensar y no solo } \\
\text { reproducir contenidos }\end{array}$ & 0,987 \\
\hline Genero y comunico el sentido de normas explicitas de convivencia & 0,986 \\
\hline Utilizo estrategias de comunicación efectivas en el aula & 0,986 \\
\hline Estructuro adecuadamente el espacio físico para la fluidez del trabajo pedagógico & 0,986 \\
\hline $\begin{array}{l}\text { Monitoreo el proceso de comprensión y apropiación del aprendizaje por parte de } \\
\text { mis estudiantes }\end{array}$ & 0,986 \\
\hline $\begin{array}{l}\text { Utilizo la información que proporciona la evaluación para identificar fortalezas } \\
\text { y debilidades en mi enseñanza }\end{array}$ & 0,986 \\
\hline $\begin{array}{l}\text { Comunico en forma apropiada y oportuna a mis estudiantes los criterios de } \\
\text { evaluación }\end{array}$ & 0,987 \\
\hline $\begin{array}{l}\text { Valoro la evaluación diagnóstica para adaptar la planificación a las necesidades } \\
\text { de mis estudiantes }\end{array}$ & 0,987 \\
\hline $\begin{array}{l}\text { Estoy preparado(a) para utilizar los resultados de la evaluación educativa con } \\
\text { el objeto de retroalimentar el aprendizaje de mis estudiantes }\end{array}$ & 0,987 \\
\hline $\begin{array}{l}\text { Selecciono estrategias e instrumentos de evaluación en función del tipo de } \\
\text { aprendizaje a lograr (conceptuales, procedimentales o actitudinales) }\end{array}$ & 0,986 \\
\hline Identifico mis fortalezas y debilidades, en relación con las disciplinas que enseño & 0,986 \\
\hline Sé cómo construir relaciones de trabajo colaborativo con mis colegas & 0,987 \\
\hline $\begin{array}{l}\text { Estoy preparado(a) para introducir cambios en la mejorara de mi práctica } \\
\text { pedagógica en función de los resultados de aprendizaje obtenidos }\end{array}$ & 0,987 \\
\hline $\begin{array}{l}\text { Analizo críticamente mi práctica pedagógica en función del impacto en el } \\
\text { aprendizaje de mis estudiantes }\end{array}$ & 0,986 \\
\hline $\begin{array}{l}\text { Tengo una sólida formación ética que me permite estar consciente de mi } \\
\text { responsabilidad en el desarrollo personal de mis estudiantes }\end{array}$ & 0,987 \\
\hline $\begin{array}{l}\text { Tengo una sólida formación ética que me permite estar consciente de mi } \\
\text { responsabilidad en el desarrollo académico de mis estudiantes }\end{array}$ & 0,986 \\
\hline $\begin{array}{l}\text { Estoy preparado(a) para ser evaluado(a) en mi quehacer pedagógico al interior } \\
\text { del aula }\end{array}$ & 0,987 \\
\hline $\begin{array}{l}\text { Conozco los marcos nacionales que regulan el desempeño y la evaluación de la } \\
\text { profesión docente }\end{array}$ & 0,987 \\
\hline $\begin{array}{l}\text { Manejo información actualizada sobre mi profesión, el sistema educativo y las } \\
\text { políticas vigentes }\end{array}$ & 0,987 \\
\hline Reconozco mis necesidades de desarrollo y actualización profesional & 0,987 \\
\hline
\end{tabular}

Como se observa, al eliminar cualquier ítem del instrumento, el indicador de consistencia interna no mejora más de lo que está e incluso disminuye levemente la confiabilidad, por lo tanto, se recomienda no quitar ningún ítem del instrumento. 


\section{Conclusiones}

El cuestionario elaborado para valorar desempeños pedagógicos en la formación inicial del profesorado cuenta con cinco dimensiones que comprenden el proceso curricular, la metodología de enseñanza aprendizaje, el ambiente para el aprendizaje, la evaluación del proceso y la reflexión pedagógica. Este instrumento cuenta con 33 ítems, con lo que es posible valorar los desempeños que cada estudiante de pedagogía debe mostrar en sus procesos de práctica.

Los resultados muestran que el instrumento presenta adecuadas características psicométricas, respecto a la validez de contenido a través de personas expertas y la consistencia interna mediante el alfa de Cronbach. Respecto de la validez total, inicialmente el instrumento comprendía 50 ítems, luego del proceso estadístico al que fueron sometidos los reactivos este quedó conformado por 33 ítems.

En relación con la confiabilidad, el instrumento mostró un coeficiente alfa de Cronbach de 0.98 . Este valor muestra una fuerte consistencia interna para un instrumento de esta naturaleza. Se observa que la consistencia en los 58 datos analizados están por sobre el 0,9 y, por lo tanto, muy cercanos al valor máximo de confiabilidad.

En general, el instrumento elaborado puede ayudar con fines de diagnóstico y de manera formativa a identificar los desempeños pedagógicos de estudiantes en procesos de formación inicial.

Finalmente, una de las ventajas del estudio que podemos mencionar es su bajo costo y la facilidad de aplicación en diversas instituciones que forman docentes, sean de educación preescolar, enseñanza básica y enseñanza media. Pues tal cual lo señalan los estándares orientadores para estudiantado egresado de carreras de pedagogía en educación básica, los desempeños pedagógicos son transversales a cualquier formación en el área pedagógica, ya que, refieren a los conocimientos, habilidades y actitudes profesionales necesarias para el desarrollo del proceso de enseñanza, que debe poseer cualquier profesional que egresa de pedagogía, de manera independiente a la disciplina que le corresponda enseñar (Ministerio de Educación, 2011).

\section{ReFERENCIAS}

Brunner, J. (2008). El proceso de Bolonia en el horizonte Latinoamericano: Límites y posibilidades. Revista de Educación Superior, número extraordinario,119-145. Recuperado de http://www.revistaeducacion.mec.es/ re2008/re2008_06.pdf

Calderhead, J. and Shorrock, S. B. (1997). Understanding Teacher Education. [Comprendiendo la formación de profesores]. London: The Falmer Press.

Castillo, S. (2002). Compromisos de la evaluación educativa. Madrid: Pearson.

Day, C. (2006). Pasión por enseñar. La identidad personal y profesional del docente y sus valores. Madrid: Narcea.

Fuentealba, R. (2003). La inserción profesional de profesores principiantes de enseñanza media (Tesis de Doctorado, inédita). Pontificia Universidad Católica de Chile. Santiago, Chile.

Galaz, A., Fuentealba, R., Cornejo, J. y Padilla, A. (2011). Estrategias reflexivas en la formación de profesores y de formadores de profesores. Santiago: Lom.

García, R. y Parra, J. (2010). Didáctica e innovación curricular. Madrid: Catarata.

Gyslin, J. (1992). Profesores: Un análisis de su identidad social. Santiago: CIDE.

Hernández, R. Fernández, C. y Baptista, P. (2010). Metodología de la investigación. México: McGrawHill.

Imbernon, F. (2001). Formación docente y profesional. Sao Paulo: Cortez.

MINEDUC-CPEIP (2008). Marco para la buena enseñanza. Chile: Ministerio de Educación.

MINEDUC-CPEIP (2011). Estándares orientadores para egresados de carreras de pedagogía en educación básica. Centro de Perfeccionamiento, Experimentación e Investigaciones Pedagógicas. Chile: Ministerio de Educación.

OCDE y BIRD/Banco Mundial. (2009). Revisión de políticas nacionales de educación: La educación superior en Chile. Santiago: Autor. 
Paquay, L., Altet, M., Charlier, Ch., y Perrenoud, P. H. (2010). La formación profesional del maestro. Estrategias y competencias. México D.F.: FCE.

Perrenoud, P. (2006). Construir competencias desde la escuela. Santiago: LOM.

Pimienta, J. (2008). Evaluación de los aprendizajes. Un enfoque basado en competencias. México: Pearson Prentice Hall.

Rovinelli, R. J., y Hambleton, R. K. (1977). On the use of content specialists in the assessment of criterion-referenced test item validity [El uso de los criterios de evaluación para validar ítems de pruebas en contenidos específicos]. Dutch Journal of Educational Research, 2, 49-60.

Saravia, M. (2009). Evaluación del profesorado universitario. Un enfoque desde la competencia profesional. USA, UK, Germany: VDM, Verlag.

Stake, R. (1967). The countenance of educational evaluation. [El rostro de la evaluación educativa]. Teacher College Record, 68, 523-540.

Tobón, S. (2007). El enfoque complejo de las competencias y el diseño curricular. Acción Pedagogía, 16, 14-28.

Tyler, R. (1986). Principios básicos del currículo. Buenos Aires: Troquel.

UNESCO. (2004). La evaluación y la acreditación en la educación superior en América Latina y el Caribe. Instituto Internacional para Educación Superior en América Latina y el Caribe (IESALC).

Yániz, C. y Villardón, L. (2006). Planificar desde competencias para promover el aprendizaje. Bilbao: Publicaciones de la Universidad de Deusto.

Zabalza, M. (2003). Competencias del profesorado universitario: Calidad y desarrollo profesional. Madrid: Narcea.

\section{BY-NC-ND}

